\title{
Chemical and Statistical Analyses of Elements in River Water of Morava e Binces
}

\author{
Fatbardh Gashi, Naser Troni, Fatmir Faiku, Fisnik Laha, \\ Arben Haziri, Ilir Kastrati, Etrit Beshtica and Mehdi Behrami
}

Department of Chemistry, Faculty of Natural Sciences, University of Pristina, Kosova

Received 2012-05-31, Revised 2012-10-31; Accepted 2013-05-13

\begin{abstract}
The objective of this research was assessment of environmental toxic elements downstream the river of Morava e Binces (Kosovo) and this study is a continuation of earlier studies of surface waters in our country. The sampling and analysis are conducted in accordance with EU and WHO standards. This study uses both quantitative and qualitative analysis to assess the water quality in several points along river. The concentration of major and minor elements was determined by Inductively Coupled Plasma Mass Spectroscopy (ICP-MS). The concentration of toxic elements which we received from surface waters are compared with the results received for the source where anthropogenic effects aren't present (the part of river in the source). The study firstly shows that Morava e Binces River is considerably polluted. Results obtained by the box plot method showed the regions with determined anomalous element concentration values in water River of Morava e Binces. This study strongly recommends the immediate correction of these issues to protect the health of population from water borne diseases.
\end{abstract}

Keywords: Chemical and Statistical Analyses, Morava e Binces, River Water, Anomalies, Pollution Assessment, ICP/MS

\section{INTRODUCTION}

Multidisciplinary collaborative research is essential for understanding the pollution processes. Determination of total quantitative and qualitative metals and distribution of all physical and chemical forms in traces (speciation) in natural water equilibrium resources today is to be considering as the main challenge for most of the scientists. Overexploitation of nature and uncontrolled use of natural resources, including inadequate processing of industrial wastes have caused large contamination of world ecosystems by toxic metals such as; $\mathrm{Hg}, \mathrm{Pb}, \mathrm{Cd}$, $\mathrm{Cu}, \mathrm{Zn}, \mathrm{Ni}, \mathrm{Mn}, \mathrm{Sb}$.

The World Health Organization (WHO) estimated that in developing countries about $80 \%$ of water pollution is a result of domestic waste. Moreover, the inadequate management of water systems can cause serious problems in the availability and quality of water (Krishnan et al., 2007). Drinking water is an essential environmental constituent and the quality of drinking water is an issue of primary interest for the residents of the European Union (Chirila et al., 2010).

Decomposition of organic matter and pollution due to anthropogenic activity are the main sources of pollution of water. Water flows freely in the active layer of water or acrotelm. Water storage is critical to the balance of water in peat swamps and at surrounding areas. Logging activity, agriculture, peat extraction and destruction of peat swamp drainage activity also give a negative effect and bad implication on the hydrology (Hamilton, 2008).

Based on the results of such studies, it will be possible in the future to propose protection and detoxification Corresponding Author: Fatbardh Gashi, Department of Chemistry, Faculty of Natural Sciences, University of Pristina, Kosova 
measures of affected river waters and general protection and remediation of ecosystems. This study is a continuation of earlier studies of surface waters in our country (Gashi et al., 2009; 2011; 2012a; 2012b).

The sampling sites in river of Morava e Binçës are geographically positioned using Geographic Information System (GIS). The results were interpreted using modern statistical methods that can be used to locate pollution sources. Selected locations, where certain toxic elements should be monitored and remediation possibly performed, were highlighted.

\section{MATERIALS AND METHODS}

\subsection{Study Area}

The aim of this study was the quantitative determination of some environmental toxic elements in the water of Morave Binçës River, as surface water resource. Morava e Binçës River, length of $49 \mathrm{~km}$, is located in south-east side of Kosova, its flow near of Gjilan city to the union of the South Morava River in Serbia. Determination of elements in water samples was performed using ICP-MS technique.

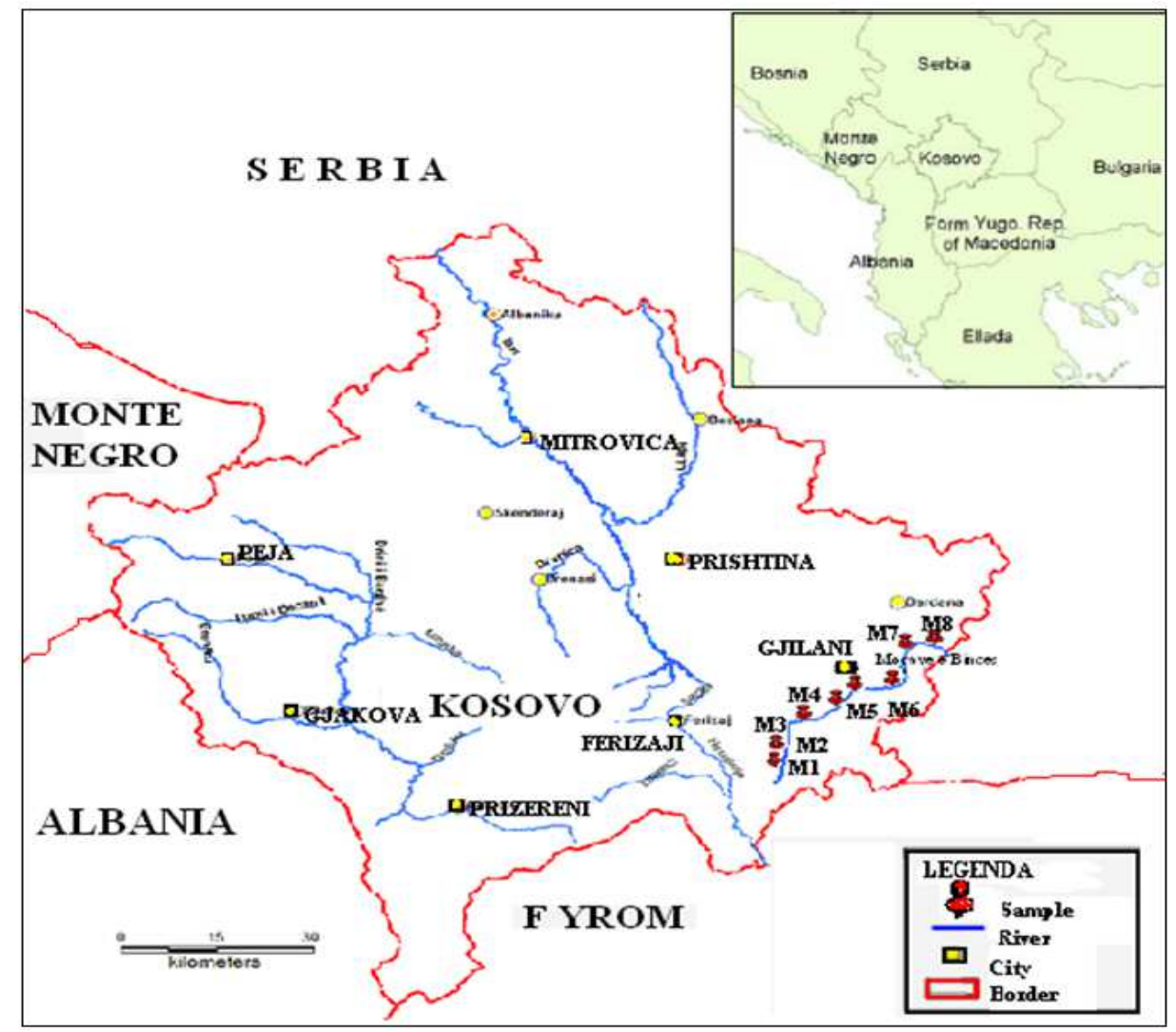

Fig. 1. Study area with sampling stations

Table 1. Sampling stations with detailed locality description

\begin{tabular}{|c|c|c|c|c|}
\hline Sample & Locality & Coordinates & Height above sea level (m) & Possible pollution sources \\
\hline$\overline{\mathrm{M}_{1}}$ & Binçë & 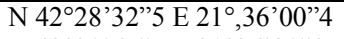 & 561 & Low probability; \\
\hline $\mathrm{M}_{2}$ & Dalja e Binçës & N 42³0’67’5 E 21³6’20’9 & 506 & Settlement; waste water; road; agriculture. \\
\hline $\mathrm{M}_{3}$ & Gjylekar & N 42³7’10”4 E 2140’05”9 & 485 & Road; agriculture; waste water from Vitia city; road. \\
\hline $\mathrm{M}_{4}$ & Partesh & N 42॰40’06”3 E 2146’77’9 & 463 & Settlement; agriculture; road. \\
\hline $\mathrm{M}_{5}$ & Uglar & N 42॰42’45’8 E 21 '53’20”6 & 452 & Settlement; road; waste water from Uglar village. \\
\hline $\mathrm{M}_{6}$ & Pogragjë & N 4243’15’0 E 2157’90’2 & 448 & Settlement; waste water from Pogragje village. \\
\hline $\mathrm{M}_{7}$ & Ranillug & N 42॰49’46”1 E 2159’39”6 & 436 & Agriculture; waste water from Gjilan city. \\
\hline $\mathrm{M}_{8}$ & Dheu i bardhë & N 4250’49”0 E 2163’35”4 & 420 & Agriculture; road \\
\hline
\end{tabular}


Our sampling strategy were concentrate in the eight monitoring points from the source in the mountain, downstream to the end of river within our territory near the border of Serbia. Surface water sampling of champions and their elaboration in the depth $\geq 0.20 \mathrm{~m}$ were done with noncontaminating bottles Pyrex according to standards methods for surface water. Some of the natural water samples are filtered with Whatman paper of $0.45 \mu \mathrm{m}$ made from cellulose nitrate in the bottle of Teflon under pressure of nitrogen (purity $99.99 \%$ ).

In natural rivers most of trace elements occur primarily as "dissolved' including colloidal species less the 0.45 or $0.4 \mu \mathrm{m}$ in these analyses filtration is avoided, to minimize contamination or losses due to adsorption. The chemical analysis of suspended particle matter itself is not done in this kind of determinations.

\subsection{Sampling and Sample Preparation}

Samples were taken from along the banks of the sampling station on 20.01.2012. Sampling tools were washed and dried with water before the next sample was collected. Water samples were collected from surface waters below $10 \mathrm{~cm}$ (Gupta, 2009). The collected samples were stored in polythene plastic containers. Weather was cloudy and rainy, with middle water levels, which was very suitable for sampling. Extraction of champions and elaboration of samples were done according to standards methods for surface water (Alper et al., 2003; APHA, 2005). The study area with the sampling locations is shown in Fig. 1 and the details about all sampling sites are presented in Table 1.

Geographical positions were determined by GPS, using model "geko 201, 12 channel". The numbers of sampling spots is 9 and in every sampling spot were taken samples in order to determine the chemical parameters. Each sampling spot of water in the river of Morava e Binçës, have been marked by codes $\mathrm{M}_{1}, \mathrm{M}_{2}$, $\mathrm{M}_{3}, \mathrm{M}_{4}, \mathrm{M}_{5}, \mathrm{M}_{6}, \mathrm{M}_{7}$ and $\mathrm{M}_{8}$.

\subsection{Chemical Characterization}

Determination of elements in water samples was performed in commercial laboratory ACTLABS, Ontario, Canada, using a "Perkin Elmer SCIEX ELAN 6100 " ICP-MS instrument, with the program "Code 6 Hydrogeochemistry".

\subsection{Statistical Methods}

Program Statistica 6.0 (Statsoft, 2001) has been used for all statistical calculations in this work, such as: determination of basic statistical parameters and determination of anomalies (extremes and outliers) for solution data. Outlier values are between 1.5 and 3 and extreme values above 3 standard deviations.

\section{RESULTS}

\subsection{ICP-MS Analyses of Major and Trace Elements}

Table 2 presents concentrations of major and trace elements $\left(\mu \mathrm{g} \mathrm{L}^{-1}\right)$ in water of river Morava e Binçës. The results for Pearson's Correlation Coefficient are displayed on Table 5.

Determination of basic statistical parameters and anomalous values of concentrations for elements: The database (Table 2) will be used in statistical calculation and in physico-chemical assessment of water quality. Table 3 presents basic statistical parameters for 58 elements in 8 water samples of Morava e Binçës River. For each element, the values are given as arithmetic mean, geometric mean, median, minimal and maximal concentration, variance, standard deviation, skewness and curtosis. Using experimental data (Table 2, obtained by ICP-MS method) and box plot approach of Tukey (1997), anomalous values (extremes and outliers) in water were determined for the whole region. Frequency Distributions of eight measured ions and Two Dimensional Scater with Plots diagrams are presented in Fig. 2 and 3. Table 4 presents anomalous values (extremes and outliers) of concentrations for particular elements $\left(\mu \mathrm{g} \mathrm{L}^{-1}\right)$.

Table 5 show classification of the polluting segments of river, based on Norwegian standards.

\section{DISCUSSION}

Chemical data presented in the Table 2, Frequency Histograms of some measured ions (Fig. 2) and Two Dimensional Scatter and Plots diagrams (Fig. 3), can be used for the assessment of water contamination by toxic elements according with WHO standards and EU directives. Chromium, zinc, manganese, nickel, cadmium, iron, arsenic and uranium generally appeared to be significantly concentrated in the River water.

The concentrations of $\mathrm{Mn}$ above $50 \mu \mathrm{gL}^{-1}$ (according EU Directives), causing significant toxic effects were found at stations $\mathrm{M}_{2}-\mathrm{M}_{8}$. The highest concentration was found at station $\mathrm{M}_{8}\left(501 \mu \mathrm{g} \mathrm{L}^{-1}\right)$.

At all locations the concentrations $\mathrm{Cr}$ in water, are below the value causing toxic effects $\left(50 \mu \mathrm{g} \mathrm{L}^{-1}\right)$.

At all other locations the concentrations of $\mathrm{Zn}$ in water are below the value causing the lowest toxic effects $\left(3000 \mu \mathrm{gL}^{-1}\right)$. The highest concentration was found at station $\mathrm{M}_{8}\left(109 \mu \mathrm{gL}^{-1}\right)$.

The concentrations of $\mathrm{Ni}$ below $20 \mu \mathrm{gL}^{-1}$, which causes the lowest toxic effects, were found at all locations $\left(\mathrm{M}_{1}-\mathrm{M}_{8}\right)$ and the highest concentration of $\mathrm{Ni}$ was found at station $\mathrm{M}_{8}\left(4.7 \mu \mathrm{gL}^{-1}\right)$, as a result of anthropogenic pollution. 
Fatbardh Gashi et al. / American Journal of Environmental Science 9 (2): 142-155, 2013

Table 2. Concentrations $\left(\mu \mathrm{g} \mathrm{L}^{-1}\right)$ of 58 elements in water of the River Morava e Binçës

\begin{tabular}{|c|c|c|c|c|c|c|c|c|}
\hline \multirow[b]{2}{*}{ Element $/ \mu \mathrm{g} / \mathrm{L}$} & \multicolumn{8}{|c|}{ Sample point } \\
\hline & $\mathrm{M}_{1}$ & $\mathrm{M}_{2}$ & $\mathrm{M}_{3}$ & $\mathrm{M}_{4}$ & $\mathrm{M}_{5}$ & $\mathrm{M}_{6}$ & $\mathrm{M}_{7}$ & $\mathrm{M}_{8}$ \\
\hline$\overline{\mathrm{Na}}$ & 3840.000 & 5540.000 & $>35000.000$ & $>35000.000$ & $>35000.000$ & $>35000.000$ & $>35000.000$ & $>35000.00$ \\
\hline $\mathrm{Li}$ & 2.000 & 2.000 & 37.000 & 43.000 & 31.000 & 36.000 & 35.000 & 26.000 \\
\hline $\mathrm{Be}$ & $<0.100$ & $<0.100$ & $<0.100$ & $<0.100$ & $<0.100$ & $<0.100$ & $<0.100$ & $<0.100$ \\
\hline $\mathrm{Mg}$ & 11100.000 & 8570.000 & 16600.000 & 19700.000 & $>20000.000$ & $>20000.000$ & $>20000.000$ & $>20000.000$ \\
\hline $\mathrm{Al}$ & 10.000 & 6.000 & 10.000 & 19.000 & 12.000 & 14.000 & 13.000 & 20.000 \\
\hline $\mathrm{Si}$ & 5600.000 & 4600.000 & 7700.000 & 8500.000 & 8700.000 & 9200.000 & 9200.000 & 9400.000 \\
\hline $\mathrm{K}$ & 1330.000 & 2120.000 & 9380.000 & 9640.000 & 10400.000 & 11100.000 & 11500.000 & 10200.000 \\
\hline $\mathrm{Ca}$ & $>20000.000$ & $>20000.000$ & $>20000.000$ & $>20000.000$ & $>20000.000$ & $>20000.000$ & $>20000.000$ & $>20000.000$ \\
\hline $\mathrm{Sc}$ & 1.000 & $<1.000$ & 1.000 & 2.000 & 2.000 & 2.000 & 2.000 & 2.000 \\
\hline $\mathrm{Ti}$ & $0,8.000$ & $0,7.000$ & $1,4.000$ & $1,6.000$ & $2,6.000$ & 3.000 & $3,2.000$ & $2,6.000$ \\
\hline V & $0,2.000$ & $0,3.000$ & $0,9.000$ & $0,9.000$ & $1,1.000$ & $1,2.000$ & $1,4.000$ & $1,2.0000$ \\
\hline $\mathrm{Cr}$ & $1,8.000$ & $1,7.000$ & $1,2.000$ & $1,5.000$ & $1,3.000$ & $1,6.000$ & 1.000 & $2,7.000$ \\
\hline $\mathrm{Mn}$ & $3,3.000$ & $22,3.000$ & 123.000 & 189.000 & 258.000 & 241.000 & 293.000 & 501.000 \\
\hline $\mathrm{Fe}$ & 60.000 & 60.000 & 250.000 & 300.000 & 330.000 & 310.000 & 330.000 & 330.000 \\
\hline Co & $0,074.000$ & $0,084.000$ & $0,226.000$ & $0,297.000$ & $0,445.000$ & $0,495.000$ & $0,512.000$ & $0,734.000$ \\
\hline $\mathrm{Ni}$ & $0,9.000$ & $0,7.000$ & $1,5.000$ & $1,3.000$ & $2,6.000$ & $3,4.000$ & $2,6.000$ & $4,7.000$ \\
\hline $\mathrm{Cu}$ & $3,4.000$ & $1,4.000$ & $2,7.000$ & $2,2.000$ & $3,1.000$ & $2,4.000$ & $2,2.000$ & $4,2.000$ \\
\hline $\mathrm{Zn}$ & $10,3.000$ & $2,5.000$ & $6,8.000$ & $5,3.000$ & $8,8.000$ & $6,8.000$ & $5,7.000$ & 109.000 \\
\hline $\mathrm{Ga}$ & $<0.010$ & $0,01.000$ & $0,01.000$ & $0,01.000$ & $0,01.000$ & $0,01.000$ & $0,02.000$ & $0,02.000$ \\
\hline Ge & $<0.010$ & $0,01.000$ & $0,34.000$ & $0,29.000$ & $0,15.000$ & $0,19.000$ & $0,14.000$ & $0,1.000$ \\
\hline As & $1,17.000$ & $0,85.000$ & $1,7.000$ & $1,76.000$ & $2,49.000$ & $2,65.000$ & $4,06.000$ & $3,32.000$ \\
\hline $\mathrm{Se}$ & $<0,2.000$ & $<0,2.000$ & $0,6.000$ & $0,7.000$ & $0,6.000$ & $0,9.000$ & 1.000 & $0,6.000$ \\
\hline $\mathrm{Rb}$ & $0,343.000$ & $0,566.000$ & $14,1.000$ & $14,1.000$ & $10,5.000$ & $11,4.000$ & $10,8.000$ & $7,33.000$ \\
\hline $\mathrm{Sr}$ & 137.000 & $>200.000$ & $>200.000$ & $>200.000$ & $>200.000$ & $>200.000$ & $>200.000$ & $>200.000$ \\
\hline $\mathrm{Y}$ & $0,025.000$ & $0,024.000$ & $0,042.000$ & $0,049.000$ & $0,04.000$ & $0,048.000$ & $0,073.000$ & $0,081.000$ \\
\hline $\mathrm{Zr}$ & $0,02.000$ & $0,01.000$ & $0,09.000$ & $0,08.000$ & $0,05.000$ & $0,05.000$ & $0,06.000$ & $0,06.000$ \\
\hline $\mathrm{Nb}$ & $<0.005$ & $<0.005$ & $<0.005$ & $<0.005$ & $0,007.000$ & $0,006.000$ & $0,007.000$ & $0,006.000$ \\
\hline Mo & $0,3.000$ & $0,3.000$ & $0,4.000$ & $0,4.000$ & $0,5.000$ & $0,7.000$ & $2,4.000$ & $0,8.000$ \\
\hline Ag & $1,3.000$ & $0,6.000$ & $0,3.000$ & $<0,2.000$ & $<0,2.000$ & $<0,2.000$ & $<0,2.000$ & $0,4.000$ \\
\hline $\mathrm{Cd}$ & $0,03.000$ & $0,04.000$ & $0,01.000$ & $<0,01.000$ & $0,46.000$ & $0,66.000$ & $0,46.000$ & $0,34.000$ \\
\hline In & $<0.001$ & $<0.001$ & $<0.001$ & $<0.001$ & $<0.001$ & $<0.001$ & $<0.001$ & $<0.001$ \\
\hline $\mathrm{Sn}$ & $<0.100$ & $<0.100$ & $<0.100$ & $<0.100$ & $<0.100$ & $<0.100$ & $<0.100$ & $<0.100$ \\
\hline $\mathrm{Sb}$ & $0,26.000$ & $0,2.000$ & $0,18.000$ & $0,16.000$ & $0,24.000$ & $0,27.000$ & $0,26.000$ & $0,46.000$ \\
\hline $\mathrm{Te}$ & $<0.100$ & $<0.100$ & $<0.100$ & $<0.100$ & $<0.100$ & $<0.100$ & $<0.100$ & $<0.100$ \\
\hline Cs & $0,002.000$ & $<0,001.000$ & $1,65.000$ & $1,19.000$ & $0,601.000$ & $0,656.000$ & $0,754.000$ & $0,393.000$ \\
\hline $\mathrm{Ba}$ & $24,9.000$ & $32,4.000$ & $58,5.000$ & $60,4.000$ & $53,6.000$ & 53.000 & $59,5.000$ & $61,8.000$ \\
\hline $\mathrm{La}$ & $0,062.000$ & $0,019.000$ & $0,032.000$ & $0,036.000$ & $0,025.000$ & $0,037.000$ & $0,043.000$ & $0,064.000$ \\
\hline $\mathrm{Ce}$ & $0,071.000$ & $0,033.000$ & $0,057.000$ & $0,064.000$ & $0,046.000$ & $0,071.000$ & $0,082.000$ & $0,137.000$ \\
\hline $\mathrm{Pr}$ & $0,006.000$ & $0,004.000$ & $0,007.000$ & $0,008.000$ & $0,006.000$ & 9.000 & $0,009.000$ & $0,015.000$ \\
\hline $\mathrm{Nd}$ & $0,017.000$ & $0,017.000$ & $0,028.000$ & $0,028.000$ & $0,026.000$ & $0,029.000$ & $0,029.000$ & $0,051.000$ \\
\hline $\mathrm{Sm}$ & $0,005.000$ & $0,004.000$ & $0,009.000$ & $0,006.000$ & $0,005.000$ & $0,007.000$ & $0,01.000$ & $0,013.000$ \\
\hline $\mathrm{Eu}$ & $0,002.000$ & $0,003.000$ & $0,006.000$ & $0,007.000$ & $0,005.000$ & $0,006.000$ & $0,006.000$ & $0,007.000$ \\
\hline Gd & $0,005.000$ & $0,002.000$ & $0,007.000$ & $0,009.000$ & $0,011.000$ & $0,017.000$ & $0,013.000$ & $0,014.000$ \\
\hline $\mathrm{Tb}$ & $<0.001$ & $<0.001$ & $0,001.000$ & $<0.001$ & $0,001.000$ & $0,001.000$ & $0,001.000$ & $0,002.000$ \\
\hline Dy & $0,003.000$ & $0,003.000$ & $0,06.000$ & $0,006.000$ & $0,006.000$ & $0,007.000$ & $0,009.000$ & $0,011.000$ \\
\hline Ho & $<0,001.000$ & $<0,001.000$ & $0,001.000$ & $0,001.000$ & $0,001.000$ & $0,002.000$ & $0,002.000$ & $0,002.000$ \\
\hline $\mathrm{Er}$ & $0,002.000$ & $0,002.000$ & $0,003.000$ & $0,003.000$ & $0,004.000$ & $0,004.000$ & $0,005.000$ & $0,006.000$ \\
\hline $\mathrm{Tm}$ & $<0.001$ & $<0.001$ & $<0.001$ & $<0.001$ & $<0.001$ & $<0.001$ & $<0.001$ & $<0.001$ \\
\hline $\mathrm{Yb}$ & $0,001.0001$ & $<0.001$ & $0,004.000$ & $0,002.000$ & $0,003.000$ & $0,003.000$ & $0,006.000$ & $0,007.000$ \\
\hline $\mathrm{Lu}$ & $<0.001$ & $<0.001$ & $<0.001$ & $<0.001$ & $<0.001$ & $<0.001$ & $0,001.000$ & $0,001.000$ \\
\hline $\mathrm{Hf}$ & $<0.001$ & $0,002.000$ & $<0.001$ & $<0.001$ & $<0.001$ & $<0.001$ & $<0.001$ & $<0.001$ \\
\hline Ta & $<0.001$ & $<0.001$ & $<0.001$ & $<0.001$ & $<0.001$ & $<0.001$ & $<0.001$ & $<0.001$ \\
\hline W & $<0.020$ & $<0.020$ & $<0.02$ & $<0.020$ & $<0.020$ & $<0.020$ & $0,02.000$ & $<0.02$ \\
\hline $\mathrm{Tl}$ & $<0.001$ & $<0.001$ & $0,005.000$ & $0,003.000$ & $0,004.000$ & $0,005.000$ & $0,006.000$ & $0,006.000$ \\
\hline $\mathrm{Pb}$ & $5,08.000$ & $5,43.000$ & $2,09.000$ & $2,9.000$ & $2,17.000$ & $3,27.000$ & $0,7.000$ & $4,22.000$ \\
\hline $\mathrm{Bi}$ & $<0.300$ & $<0.300$ & $<0.300$ & $<0.300$ & $<0.300$ & $<0.300$ & $<0.300$ & $<0.300$ \\
\hline Th & $<0.001$ & $<0.001$ & $<0.001$ & $<0.001$ & $<0.001$ & $0,001.000$ & $0,003.000$ & $0,003.000$ \\
\hline U & $0,334.000$ & $0,338.000$ & $1,72.000$ & $1,82.000$ & $1,86.000$ & $2,01.00$ & $2,16.000$ & $2,22.000$ \\
\hline
\end{tabular}


Fatbardh Gashi et al. / American Journal of Environmental Science 9 (2): 142-155, 2013

Table 3. Basic statistical parameters for 32 elements $\left(\mu \mathrm{g} \mathrm{L}^{-1}\right)$ in 8 water samples

\begin{tabular}{|c|c|c|c|c|c|c|c|c|c|c|}
\hline \multirow{2}{*}{$\begin{array}{l}\text { Element } \\
/ \mu \mathrm{g} \mathrm{L}^{-1}\end{array}$} & \multicolumn{10}{|c|}{ Basic statistical parameters } \\
\hline & Valid N & Mean & Geometric & c Median & Minimum & Maximum & Variance & Std. Dev. & Skewness & Kurtosis \\
\hline $\mathrm{Li}$ & 8 & 26.500 & 16.839 & 33.000 & 2.000 & 43.00 & 252 & 15.884 & -1.05525 & -0.47935 \\
\hline $\mathrm{Al}$ & 8 & 13.000 & 12.222 & 12.500 & 6.000 & 20.00 & 22 & 4.690 & 0.29903 & -0.46411 \\
\hline $\mathrm{Si}$ & 8 & 7862.500 & $7645.128 \quad 8$ & 8600.000 & 4600.000 & 9400.00 & 3262679 & 1806.289 & -1.19732 & 0.00021 \\
\hline $\mathrm{K}$ & 8 & 8208.750 & 6565.4619 & 9920.000 & 1330.000 & 11500.00 & 16538384 & 4066.741 & -1.32663 & -0.07978 \\
\hline $\mathrm{Ti}$ & 8 & 1.988 & 1.733 & 2.100 & 0.700 & 3.20 & 1 & 0.986 & -0.15679 & -1.86614 \\
\hline $\mathrm{V}$ & 8 & 0.900 & 0.757 & 1.000 & 0.200 & 1.40 & 0 & 0.434 & -0.86531 & -0.56116 \\
\hline $\mathrm{Cr}$ & 8 & 1.600 & 1.536 & 1.550 & 1.000 & 2.70 & 0 & 0.518 & 1.41231 & 2.84405 \\
\hline $\mathrm{Mn}$ & 8 & 203.825 & 105.728 & 215.000 & 3.300 & 501.00 & 25785 & 160.578 & 0.57909 & 0.57557 \\
\hline $\mathrm{Fe}$ & 8 & 246.250 & 204.072 & 305.000 & 60.000 & 330.00 & 13912 & 117.951 & -1.24721 & -0.33150 \\
\hline $\mathrm{Co}$ & 8 & 0.358 & 0.277 & 0.371 & 0.074 & 0.73 & 0 & 0.229 & 0.21497 & -0.76374 \\
\hline $\mathrm{Ni}$ & 8 & 2.213 & 1.842 & 2.050 & 0.700 & 4.70 & 2 & 1.376 & 0.75640 & -0.16495 \\
\hline $\mathrm{Cu}$ & 8 & 2.700 & 2.577 & 2.550 & 1.400 & 4.20 & 1 & 0.860 & 0.39138 & 0.30760 \\
\hline $\mathrm{Zn}$ & 8 & 19.400 & 8.755 & 6.800 & 2.500 & 109.00 & 1316 & 36.279 & 2.80513 & 7.90161 \\
\hline As & 8 & 2.250 & 2.008 & 2.125 & 0.850 & 4.06 & 1 & 1.089 & 0.43385 & -0.65868 \\
\hline $\mathrm{Se}$ & 8 & 0.600 & 0.521 & 0.600 & 0.200 & 1.00 & 0 & 0.288 & -0.28751 & -0.65505 \\
\hline $\mathrm{Rb}$ & 8 & 8.642 & 4.959 & 10.650 & 0.343 & 14.10 & 30 & 5.492 & -0.84895 & -0.80337 \\
\hline $\mathrm{Y}$ & 8 & 0.048 & 0.044 & 0.045 & 0.024 & 0.08 & 0 & 0.020 & 0.60455 & -0.52532 \\
\hline $\mathrm{Zr}$ & 8 & 0.052 & 0.044 & 0.055 & 0.010 & 0.09 & 0 & 0.027 & -0.35078 & -0.50780 \\
\hline Mo & 8 & 0.725 & 0.560 & 0.450 & 0.300 & 2.40 & 0 & 0.701 & 2.47480 & 6.43603 \\
\hline $\mathrm{Sb}$ & 8 & 0.254 & 0.242 & 0.250 & 0.160 & 0.46 & 0 & 0.093 & 1.76683 & 4.02679 \\
\hline $\mathrm{Ba}$ & 8 & 50.513 & 48.332 & 56.050 & 24.900 & 61.80 & 196 & 13.987 & -1.32655 & 0.24563 \\
\hline $\mathrm{La}$ & 8 & 0.040 & 0.037 & 0.036 & 0.019 & 0.06 & 0 & 0.016 & 0.57208 & -0.71865 \\
\hline $\mathrm{Ce}$ & 8 & 0.070 & 0.065 & 0.068 & 0.033 & 0.14 & 0 & 0.031 & 1.47109 & 3.23477 \\
\hline $\operatorname{Pr}$ & 8 & 0.008 & 0.007 & 0.007 & 0.004 & 0.01 & 0 & 0.003 & 1.40563 & 2.92992 \\
\hline $\mathrm{Nd}$ & 8 & 0.028 & 0.027 & 0.028 & 0.017 & 0.05 & 0 & 0.011 & 1.50007 & 3.57507 \\
\hline $\mathrm{Sm}$ & 8 & 0.007 & 0.007 & 0.007 & 0.004 & 0.01 & 0 & 0.003 & 0.87402 & -0.05085 \\
\hline $\mathrm{Eu}$ & 8 & 0.005 & 0.005 & 0.006 & 0.002 & 0.01 & 0 & 0.002 & -1.04510 & -0.12675 \\
\hline $\mathrm{Gd}$ & 8 & 0.010 & 0.008 & 0.010 & 0.002 & 0.02 & 0 & 0.005 & -0.15398 & -0.78283 \\
\hline Dy & 8 & 0.006 & 0.006 & 0.006 & 0.003 & 0.01 & 0 & 0.003 & 0.37973 & -0.12571 \\
\hline $\mathrm{Er}$ & 8 & 0.004 & 0.003 & 0.004 & 0.002 & 0.01 & 0 & 0.001 & 0.47992 & -0.56450 \\
\hline $\mathrm{Pb}$ & 8 & 3.233 & 2.774 & 3.085 & 0.700 & 5.43 & 3 & 1.611 & -0.04958 & -0.83004 \\
\hline U & 8 & 1.558 & 1.260 & 1.840 & 0.334 & 2.22 & 1 & 0.773 & -1.23779 & -0.24507 \\
\hline
\end{tabular}

Table 4. Water samples with anomalous values (extremes and outliers) of concentrations for particular elements $\left(\mu \mathrm{g} \mathrm{L}^{-1}\right)$ determined in water

\begin{tabular}{lll}
\hline Sample & Extremes of elements $(®)\left(\mu \mathrm{g} \mathrm{dm}^{-3}\right)$ & Outliers of elements $\left.(\mathrm{o})(\mu \mathrm{g} \mathrm{dm})^{-3}\right)$ \\
\hline $\mathrm{M}_{1}$ & - & - \\
$\mathrm{M}_{2}$ & - & - \\
$\mathrm{M}_{3}$ & - & $\mathrm{Ni}(19.5)$ \\
$\mathrm{M}_{5}$ & - & - \\
$\mathrm{M}_{6}$ & $\mathrm{~Pb}(11.2)$ & - \\
$\mathrm{M}_{7}$ & - & - \\
$\mathrm{M}_{8}$ & $\mathrm{Zn}(109.0)$ & \\
\hline
\end{tabular}

Table 5. Correlation factors for 15 elements $\left(\mu \mathrm{g} \mathrm{L}^{-1}\right)$ in 8 water samples

\begin{tabular}{|c|c|c|c|c|c|c|c|c|c|c|c|c|c|c|c|}
\hline \multirow[b]{2}{*}{ Variable } & \multicolumn{15}{|c|}{ Correlations Marked correlations are significant at $\mathrm{p}<0.05000 \mathrm{~N}=8$ (Case wise deletion of missing data) } \\
\hline & $\mathrm{Ti}$ & V & $\mathrm{Cr}$ & $\mathrm{Mn}$ & $\mathrm{Fe}$ & $\mathrm{Co}$ & $\mathrm{Ni}$ & $\mathrm{Cu}$ & $\mathrm{Zn}$ & As & Mo & $\mathrm{Sb}$ & $\mathrm{Ba}$ & $\mathrm{Pb}$ & $\mathrm{U}$ \\
\hline$\overline{\mathrm{Ti}}$ & 1.00 & & & & & & & & & & & & & & \\
\hline V & 0.94 & 1.00 & & & & & & & & & & & & & \\
\hline $\mathrm{Cr}$ & -0.09 & -0.15 & 1.00 & & & & & & & & & & & & \\
\hline $\mathrm{Mn}$ & 0.80 & 0.83 & 0.40 & 1.00 & & & & & & & & & & & \\
\hline $\mathrm{Fe}$ & 0.87 & 0.96 & -0.11 & 0.82 & 1.00 & & & & & & & & & & \\
\hline $\mathrm{Co}$ & 0.88 & 0.88 & 0.32 & 0.98 & 0.85 & 1.00 & & & & & & & & & \\
\hline $\mathrm{Ni}$ & 0.81 & 0.77 & 0.47 & 0.94 & 0.72 & 0.96 & 1.00 & & & & & & & & \\
\hline $\mathrm{Cu}$ & 0.22 & 0.18 & 0.61 & 0.53 & 0.26 & 0.48 & 0.58 & 1.00 & & & & & & & \\
\hline $\mathrm{Zn}$ & 0.26 & 0.28 & 0.86 & 0.75 & 0.29 & 0.66 & 0.74 & 0.75 & 1.00 & & & & & & \\
\hline As & 0.93 & 0.90 & -0.02 & 0.84 & 0.80 & 0.89 & 0.79 & 0.30 & 0.40 & 1.00 & & & & & \\
\hline Mo & 0.68 & 0.64 & -0.32 & 0.45 & 0.46 & 0.50 & 0.36 & -0.09 & 0.03 & 0.83 & 1.00 & & & & \\
\hline $\mathrm{Sb}$ & 0.44 & 0.34 & 0.78 & 0.74 & 0.28 & 0.73 & 0.83 & 0.75 & 0.91 & 0.55 & 0.23 & 1.00 & & & \\
\hline $\mathrm{Ba}$ & 0.70 & 0.89 & -0.10 & 0.77 & 0.94 & 0.75 & 0.61 & 0.16 & 0.32 & 0.69 & 0.41 & 0.19 & 1.00 & & \\
\hline $\mathrm{Pb}$ & -0.66 & -0.77 & 0.68 & -0.36 & -0.75 & -0.41 & -0.23 & 0.09 & 0.24 & -0.65 & -0.65 & 0.20 & -0.72 & 1.00 & \\
\hline U & 0.86 & 0.97 & -0.06 & 0.84 & 0.99 & 0.87 & 0.76 & 0.27 & 0.35 & 0.83 & 0.50 & 0.33 & 0.96 & -0.73 & 1.00 \\
\hline
\end{tabular}


Fatbardh Gashi et al. / American Journal of Environmental Science 9 (2): 142-155, 2013

Table 6. Classification waters segments of River, based on Norwegian standards

\begin{tabular}{lllll}
\hline Element & Water class I & Water class II & Water class III & Water class IV \\
\hline $\mathrm{Zn}\left(\mu \mathrm{gL} L^{-1}\right)$ & $<30$ & $30-60$ & $60-300$ & $>300$ \\
& $\mathrm{M}_{1}-\mathrm{M}_{7}$ & - & $\mathrm{M}_{8}$ & - \\
$\mathrm{Cd}\left(\mu \mathrm{gL}^{-1}\right)$ & $<0.2$ & $0.2-0.5$ & $0.5-1$ & $>1$ \\
& $\mathrm{M}_{3} \mathrm{M}_{4}$ & $\mathrm{M}_{1} \mathrm{M}_{2} \mathrm{M}_{5} \mathrm{M}_{7} \mathrm{M}_{8}$ & $\mathrm{M}_{6}$ & \\
$\mathrm{~Pb}\left(\mu \mathrm{gL}^{-1}\right)$ & $<1$ & $1-5$ & $5-15$ & $15-40$ \\
$\mathrm{Cu}\left(\mu \mathrm{gL}^{-1}\right)$ & - & $\mathrm{M}_{3}-\mathrm{M}_{8}$ & $\mathrm{M}_{1} \mathrm{M}_{2}$ & - \\
& $<3$ & $3-15$ & $15-60$ & $>60$ \\
& $\mathrm{M}_{2}-\mathrm{M}_{4} \mathrm{M}_{6} \mathrm{M}_{7}$ & $\mathrm{M}_{1} \mathrm{M}_{5} \mathrm{M}_{8}$ & - & - \\
\hline
\end{tabular}
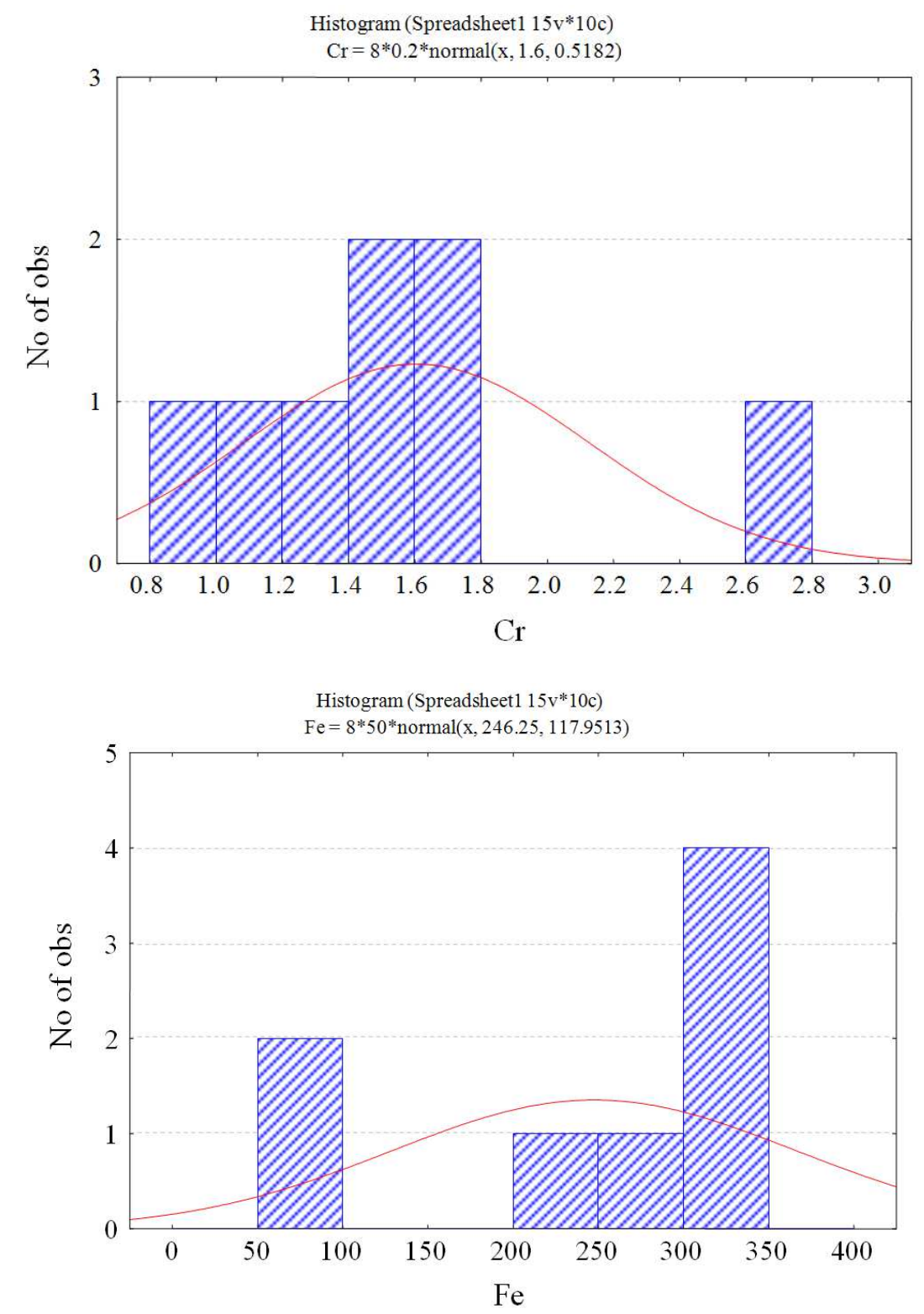

Science Publications 
Fatbardh Gashi et al. / American Journal of Environmental Science 9 (2): 142-155, 2013
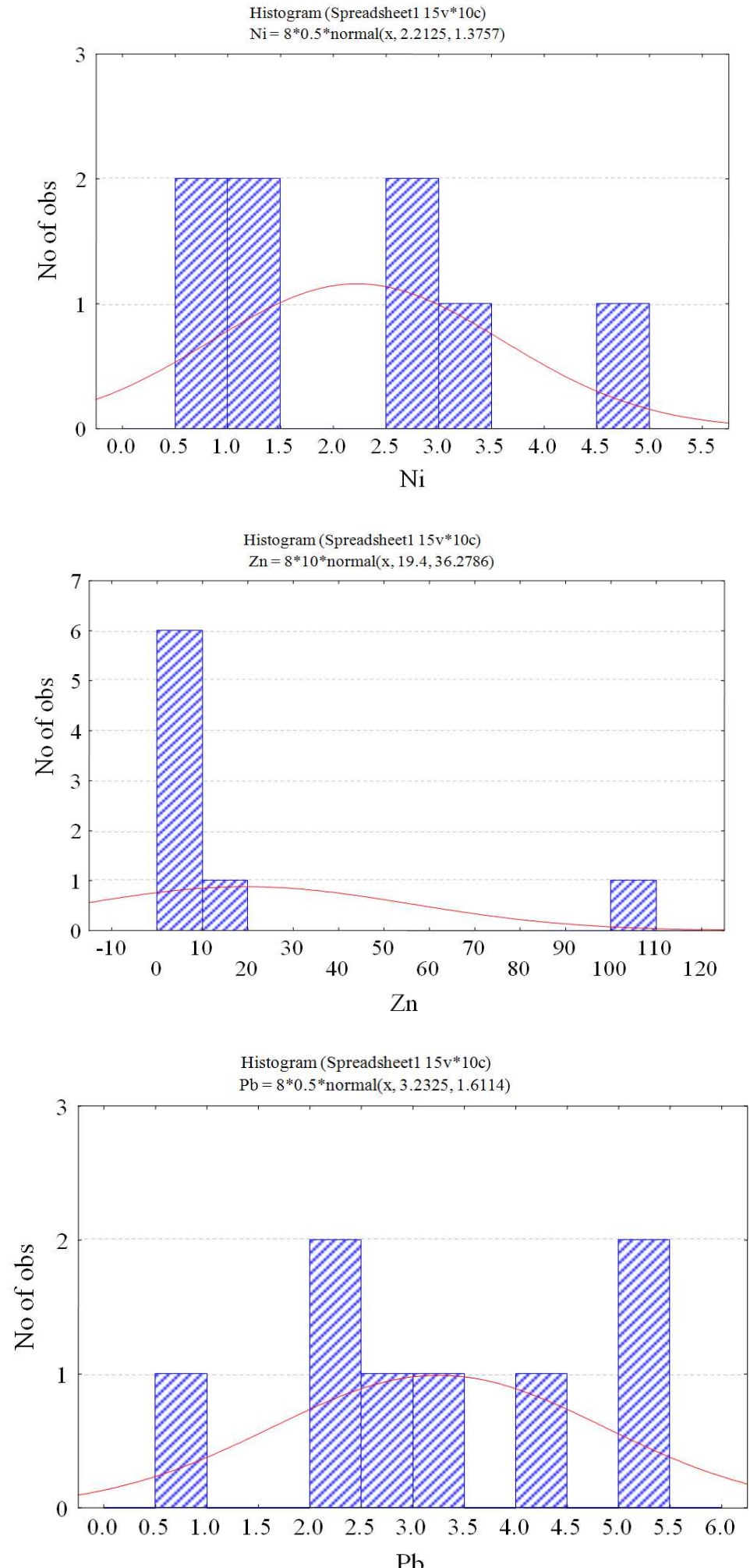

$\mathrm{Pb}$ 
Fatbardh Gashi et al. / American Journal of Environmental Science 9 (2): 142-155, 2013
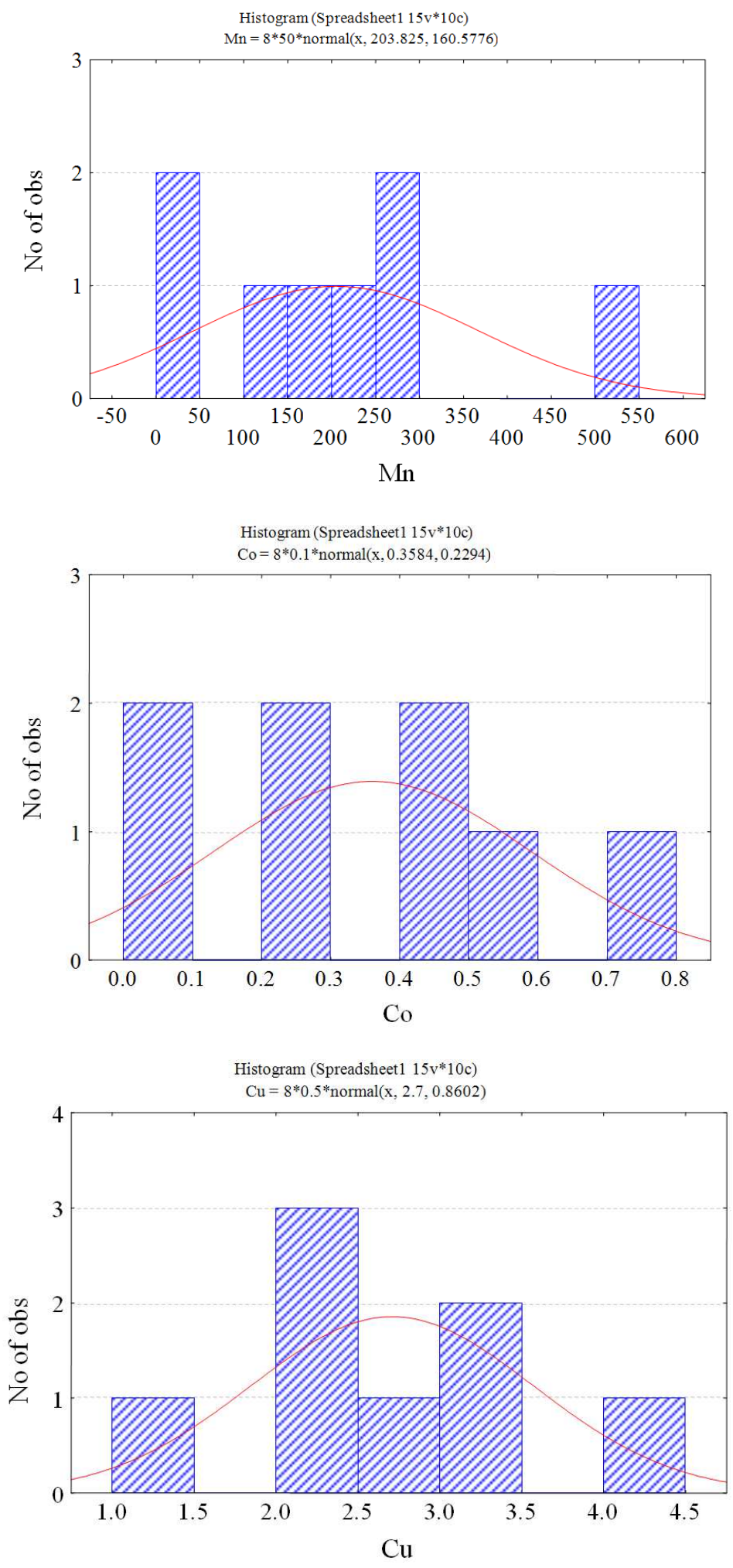
Fatbardh Gashi et al. / American Journal of Environmental Science 9 (2): 142-155, 2013
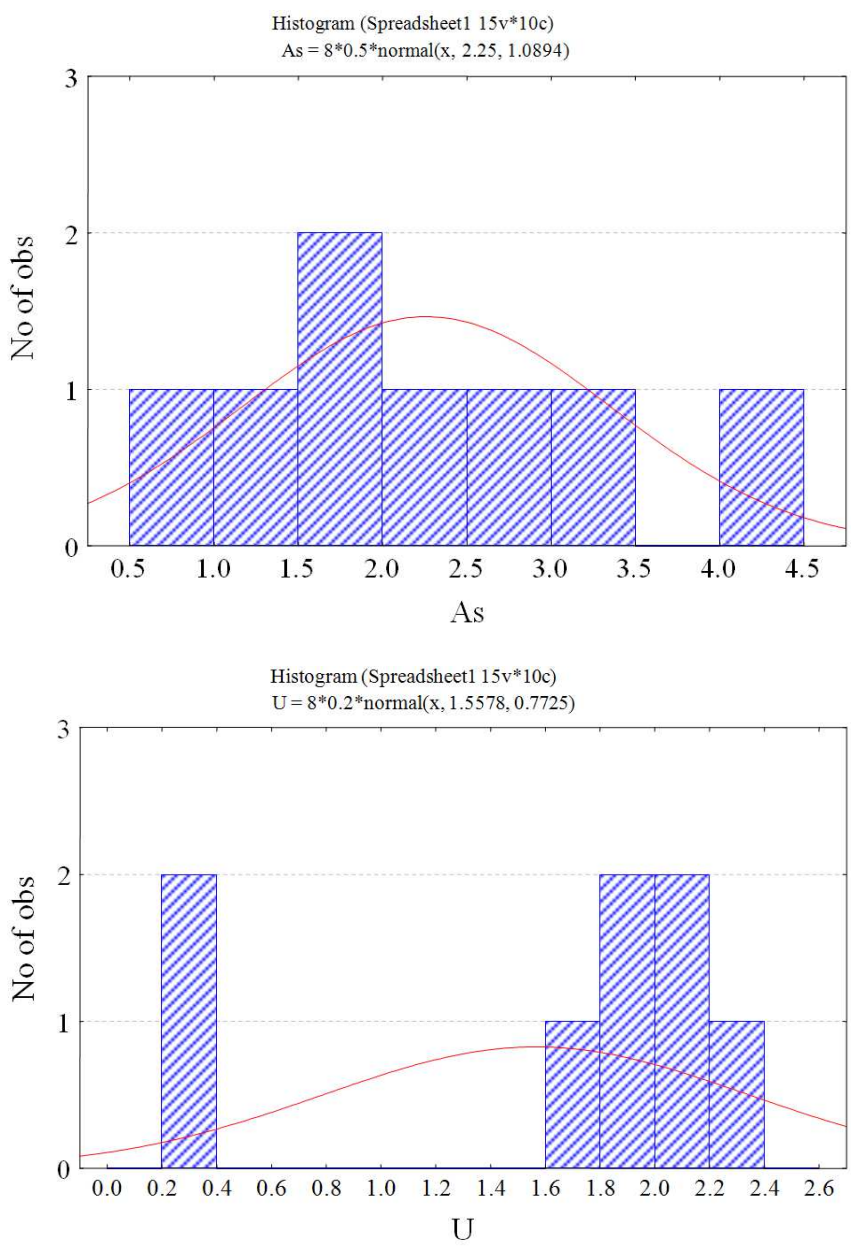

Fig. 2. Frequency histograms of some measured ions

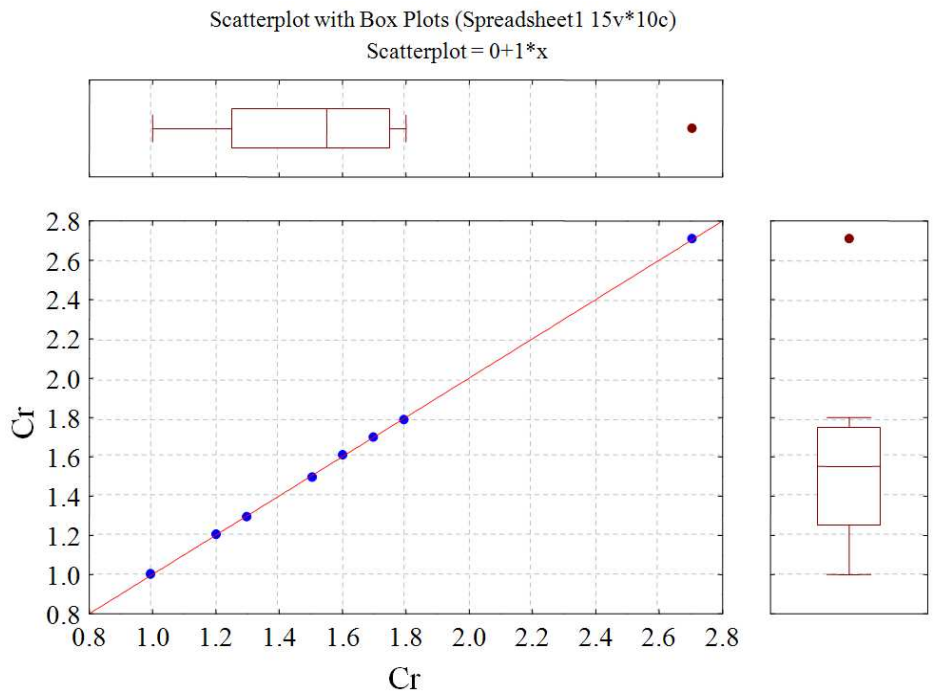


Fatbardh Gashi et al. / American Journal of Environmental Science 9 (2): 142-155, 2013
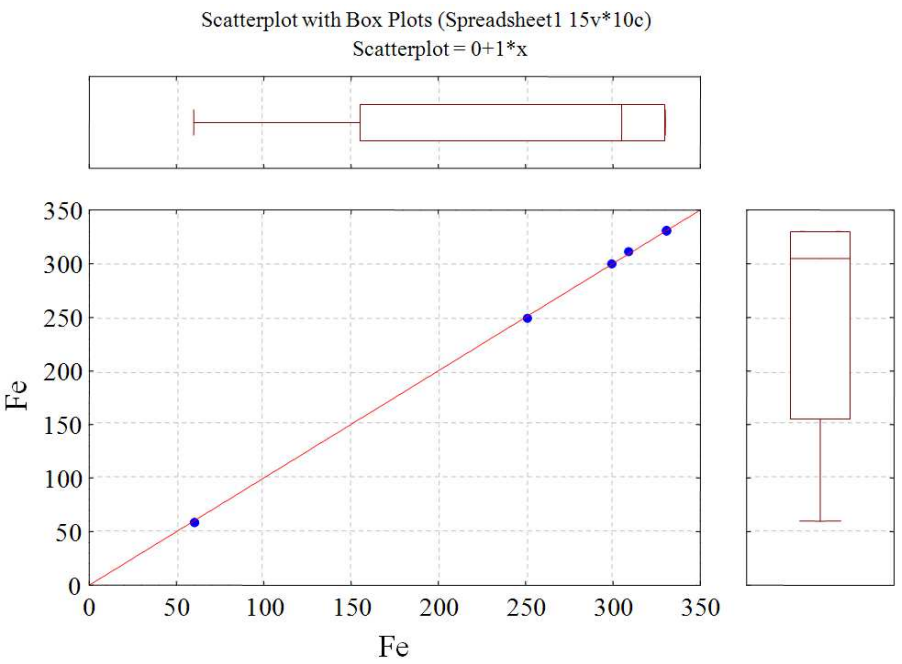

Scatterplot with Box Plots (Spreadsheet1 15v*10c) Scatterplot $=0+1 * \mathrm{x}$
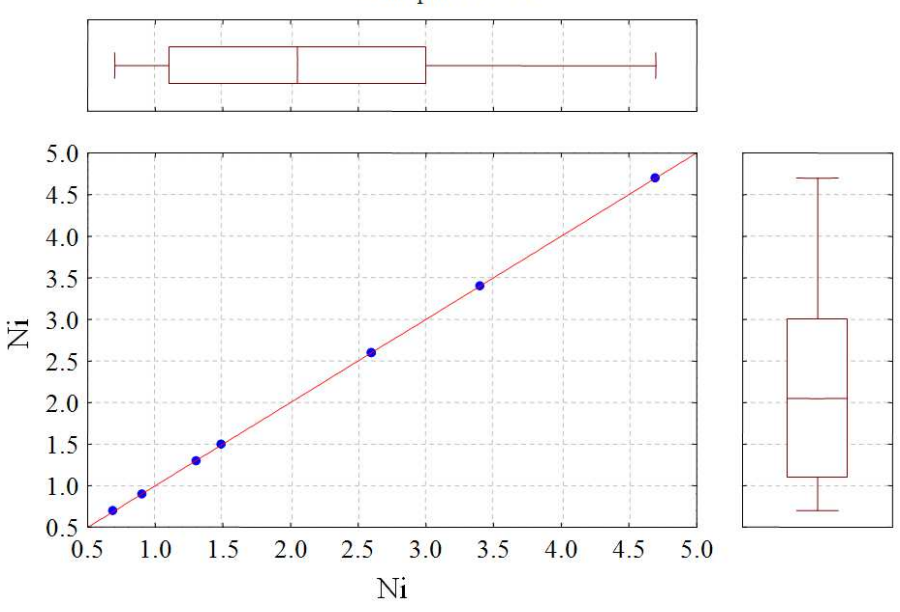

Scatterplot with Box Plots (Spreadsheet1 15v*10c) Scatterplot $=0+1 * x$
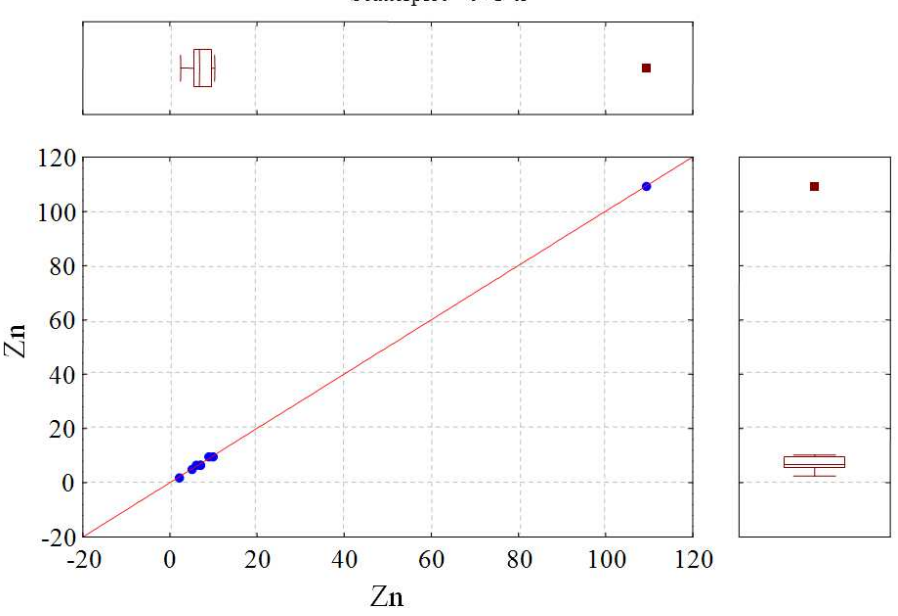
Fatbardh Gashi et al. / American Journal of Environmental Science 9 (2): 142-155, 2013
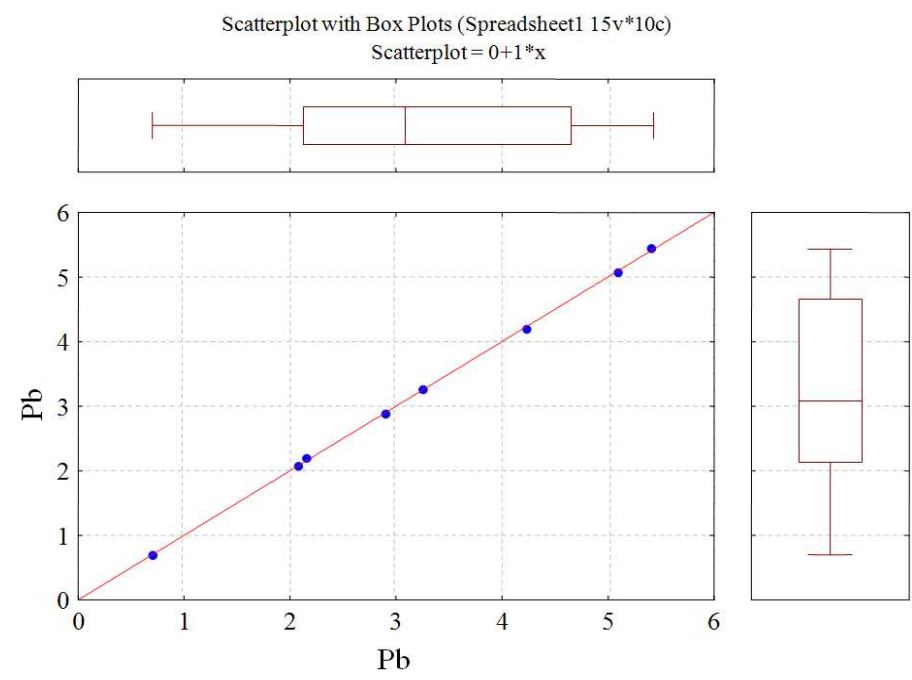

Scatterplot with Box Plots (Spreadsheet1 10v*18c) Scatterplot $=0+1 * x$
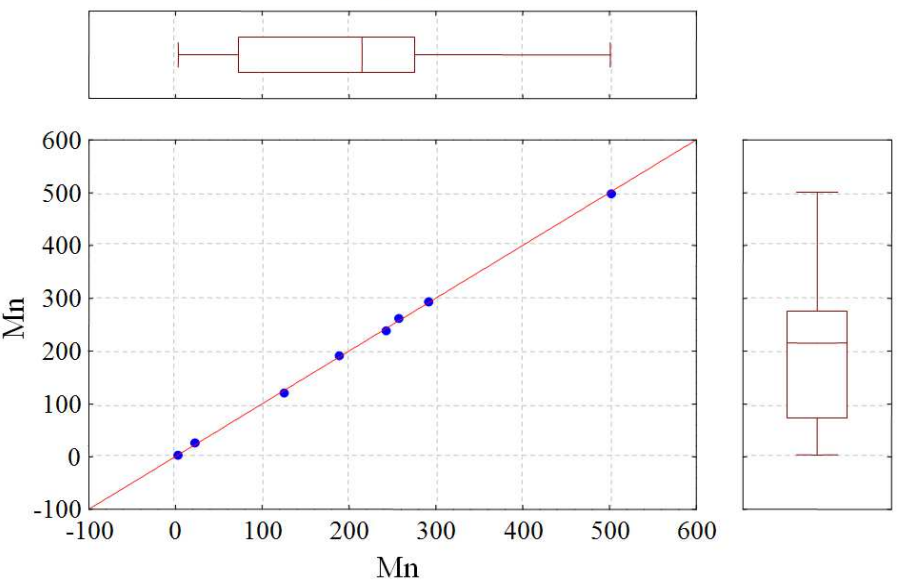

Scatterplot with Box Plots (Spreadsheet1 15v*10c) Scatterplot $=0+1^{*} \mathrm{x}$
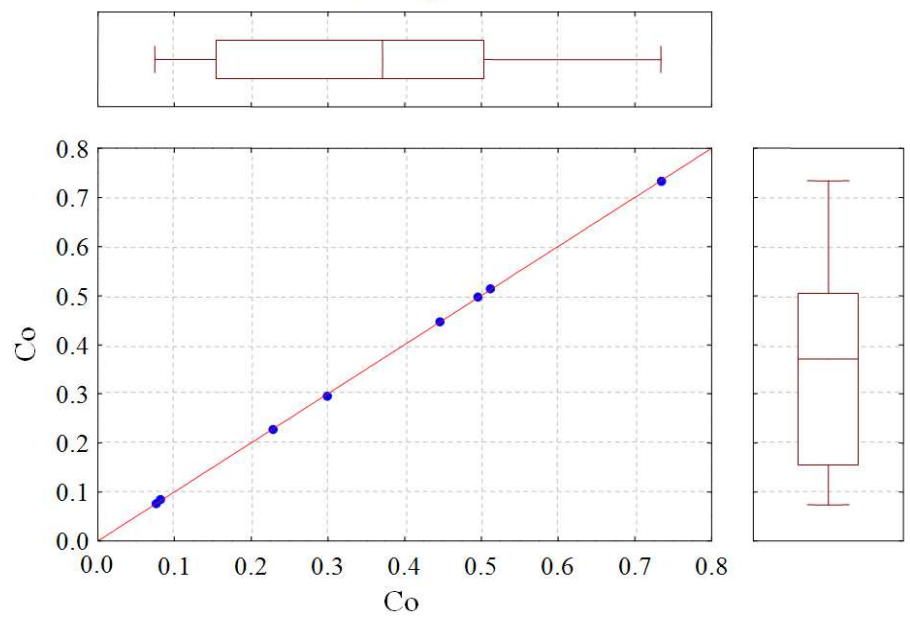
Fatbardh Gashi et al. / American Journal of Environmental Science 9 (2): 142-155, 2013
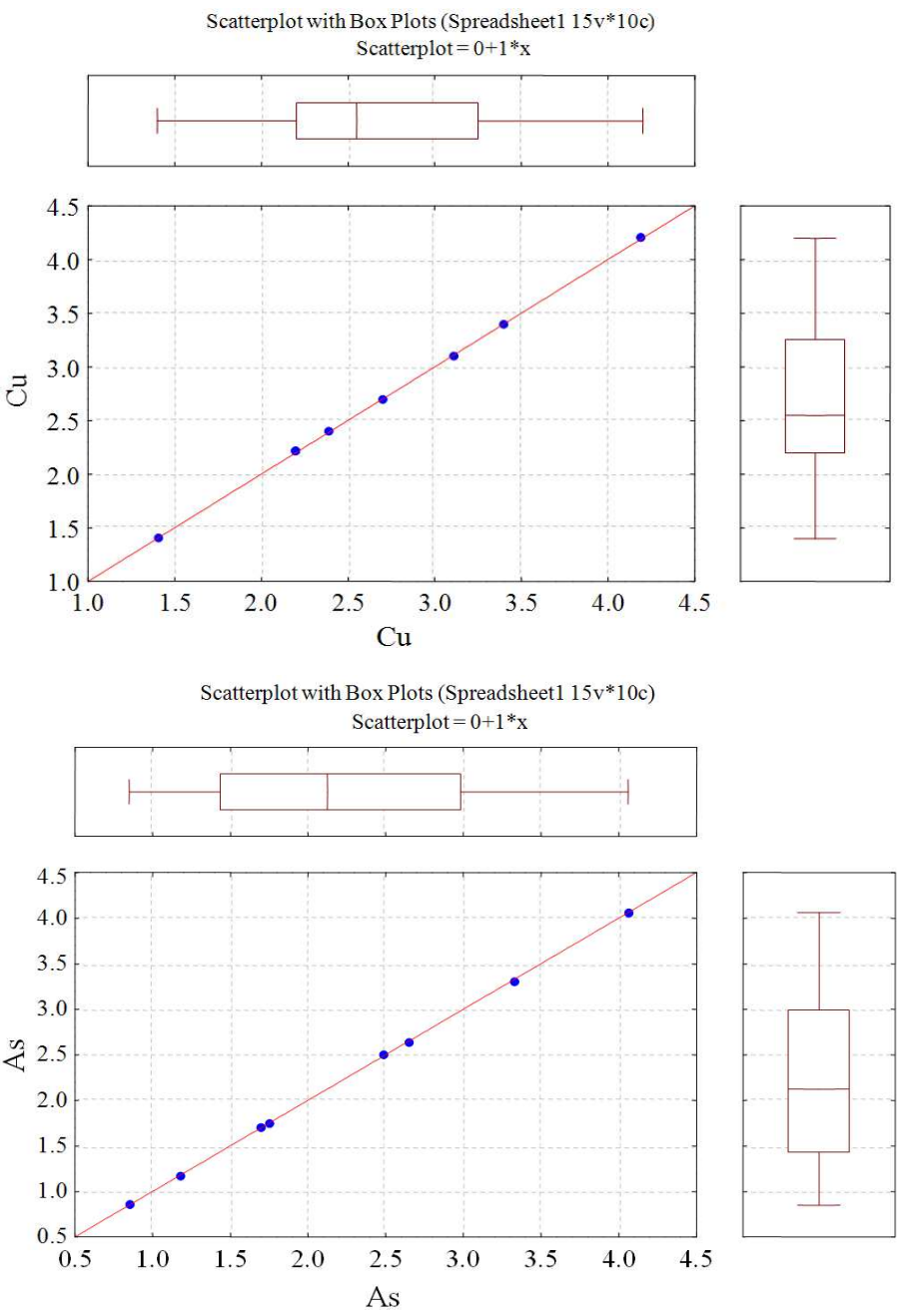

Scatterplot with Box Plots (Spreadsheet1 15v*10c) Scatterplot $=0+1 * x$
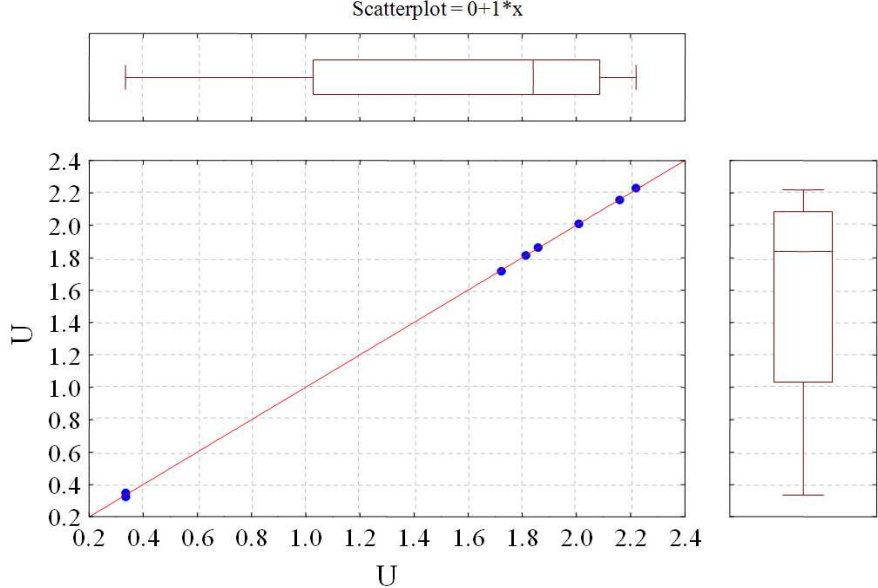

Fig. 3. Two dimensional scatter with plots diagrams of some measured ions 
The concentrations of $\mathrm{Cd}$, which causes the lowest toxic effects, were found at all locations $M_{1}-M_{8}$. The significant concentration of $\mathrm{Cd}$ was found at stations $\mathrm{M}_{5}$ $\mathrm{M}_{8}$, as a result of anthropogenic pollution.

The concentrations of as below $10 \mu \mathrm{g} \mathrm{dm}^{-3}$ which causes the lowest toxic effects, were found at all locations. The highest concentration of as was found at station $\mathrm{M}_{7}\left(4.06 \mu \mathrm{gL}^{-1}\right)$.

The concentrations of $\mathrm{Pb}$ below $10 \mu \mathrm{gL}^{-1}$ which causes the lowest toxic effects, were found at all locations. The highest concentration of $\mathrm{Pb}$ was found at station $\mathrm{M}_{2}\left(5.43 \mu \mathrm{gL}^{-1}\right)$.

The concentration of $\mathrm{Fe}$ above $300 \mu \mathrm{gL}^{-1}$, which causes the significant toxic effects, was found at stations $\mathrm{M}_{4}-\mathrm{M}_{8}$ and $\mathrm{M}_{8}\left(330 \mu \mathrm{gL}^{-1}\right)$. The highest concentration of $\mathrm{Fe}$ in water was found at station $\mathrm{M}_{5}, \mathrm{M}_{7}$ and $\mathrm{M}_{8}\left(330 \mu \mathrm{gL}^{-1}\right)$.

The concentrations of $\mathrm{U}$ below $10 \mu \mathrm{gL}^{\square 1}$ which causes the lowest toxic effects, were found at all locations. The highest concentration of $U$ was found at station $\mathrm{M}_{8}\left(2.22 \mu \mathrm{gL}^{-1}\right)$.

The results for Pearson's Correlation Coefficient displayed on Table, showed that $\mathrm{Cr}$ was an excellent and very high positive relationship with $\mathrm{Zn}, \mathrm{Sb}$ and $\mathrm{Pb}$. No correlation was found with $\mathrm{Fe}$, As, Mo, Ba and U. Mn was an excellent and very high positive relationship with Fe, Co, Ni, Zn, As, Sb and U. No correlation was found with $\mathrm{Pb}$. Fe was an excellent and very high positive relationship with $\mathrm{Co}, \mathrm{Ni}, \mathrm{As}, \mathrm{Ba}$ and $\mathrm{U}$. No correlation was found with $\mathrm{Pb}$. Co was an excellent and very high positive relationship with $\mathrm{Ni}$, Ass, $\mathrm{Sb}, \mathrm{Ba}$ and $\mathrm{U}$. No correlation was found with $\mathrm{Pb}$. $\mathrm{Ni}$ was an excellent and very high positive relationship with $\mathrm{Zn}, \mathrm{As}, \mathrm{Sb}$ and $\mathrm{U}$. No correlation was found with $\mathrm{Pb}$. $\mathrm{Cu}$ was an excellent and very high positive relationship with $\mathrm{Zn}$ and $\mathrm{Sb}$. No correlation was found with $\mathrm{Sb}$. $\mathrm{Zn}$ was an excellent and very high positive relationship with $\mathrm{Sb}$. As was an excellent and very high positive relationship with Mo and $\mathrm{U}$. No correlation was found with $\mathrm{Pb}$. $\mathrm{Ba}$ was an excellent and very high positive relationship with U. No correlation was found with $\mathrm{Pb}$. $\mathrm{BP}$ was a very high negative relationship with $\mathrm{U}$.

The River Morava e Binçës is significantly polluted with $\mathrm{Cr}, \mathrm{Zn}, \mathrm{Mn}, \mathrm{As}, \mathrm{Fe}$ and $\mathrm{U}$ as a result of natural pollution. The low levels of anthropogenic pollutions with $\mathrm{Ni}$ and $\mathrm{Cd}$ (segment $\mathrm{M}_{5}-\mathrm{M}_{8}$ ), are coming from waste waters of "Ni-Cd Accu" factory in Gjilan city.

Based on Norwegian standards of the water quality (Table 6) according the mass concentration of $\mathrm{Zn}$ (II) in the monitoring point $\mathrm{M}_{8}$, is classified in class III. Also from mass concentration of $\mathrm{Cd}$ (II) we see that monitorng point $\mathrm{M}_{6}$ is classified in water class III. Similiarly the amount of mass concentration of $\mathrm{Pb}$ (II) in the monitoring points $\mathrm{M}_{1}$ and $\mathrm{M}_{2}$ are classified in water class III. Based in the amount of mass concentration of $\mathrm{Cu}$ (II) in the monitoring points $\mathrm{M}_{1}, \mathrm{M}_{5}$ and $\mathrm{M}_{8}$ are classified in water class II.

\section{CONCLUSION}

According to the performed chemical analyses, we have noticed that water of River Morava e Binçës is significantly polluted with $\mathrm{Cr}, \mathrm{Zn}, \mathrm{Mn}, \mathrm{As}, \mathrm{Fe}, \mathrm{Pb}$ and $\mathrm{U}$, as a result of natural pollution and the low levels of pollutions of $\mathrm{Ni}$ and $\mathrm{Cd}$ as a result of anthropogenic pollution. Based on Statistical methods and norwegian standards according Table 6, for most metal indicators segments waters are classified mainly from I to III category.

\section{ACKNOWLEDGEMENT}

This study is a part of Msc. Thesis of Etrit Beshtica (supervisor Dr. Fatbardh Gashi, from the Department of Chemistry, Faculty of Natural Sciences, University of Prishtina, Kosova). Colleges from the Department of Chemistry and Geography, University of Prishtina are thanked for his assistances.

\section{REFERENCES}

Alper, B., K. Abidin and K.B. Yuksel, 2003. The effect of Yatagan thermal power plant (Mugla, Turkey) on the quality of surface and ground waters. Water Air Soil Pollut., 149: 93-111. DOI: 10.1023/A:1025660629875

APHA, 2005. Standard Methods for the Examination of Water and Wastewater. 21st Ed, American Public Health Association, Washington, DC., ISBN-10: 0875530478, pp: 1200.

Chirila, E., T. Bari and L. Barbes, 2010. Drinking water quality assessment in constanta town. Ovidius Univ. Ann. Chem., 21: 87-90.

Gashi, F, F. Faiku, A. Haziri, R. Hoti, F. Jusufi, F. Laha, B. Shala, F. Feka and A. Dreshaj, 2012b. Study of anthropogenic impact in water quality of drini $\mathrm{i}$ bardhë river (kosova). J. Int. Environ. Appli. Sci., 7: 530-537.

Gashi, F., F. Faiku, A. Haziri, R. Hoti and F. Laha et al., 2012a. Study of physical and chemical parameters in river water of lumbardh (Kosova). J. Int. Environ. Appli. Sci., 7: 373-380. 
Gashi, F., S. Franciskovic-Bilinski and H. Bilinski, 2009. Analysis of sediments of the four main rivers (drini i bardhë, Morava e Binces, lepenc and sitnica) in kosovo. Fresenius Environ. Bull., 18: 1462-1471.

Gashi, F., S. Franciskovic-Bilinski, H. Bilinski, N. Troni and M. Bacaj, 2011. Establishing of monitoring network on Kosovo Rivers: preliminary measurements on the four main rivers (Drini $\mathrm{i}$ Bardhë, Morava e Binqës, Lepenc and Sitnica). Environ. Monit. Assess., 175: 279-289. PMID: 20512619

Gupta, P.K., 2009. Methods in Environmental Analysis Water, Soil and Air. 1st Edn., Agrobios, India, ISBN-10: 8177540556, pp: 443.
Hamilton, L.S., 2008. Forests and Water: A Thematic Study Prepared in the Framework of the Global Forest Resources Assessment. 1st Edn., Food and Agriculture Organization of the United Nation, Rome, ISBN-10: 9251060908, pp: 78.

Krishnan, R.R., K. Dharmaraj and B.D.R. Kumari, 2007. A comparative study on the physicochemical and bacterial analysis of drinking, borewell and sewage water in the three different places of Sivakasi. J. En. Biol., 28: 105-108. PMID: 17717994

Statsoft, 2001. Statistica (data analysis software system), version 6.

Tukey, J.W., 1977. Exploratory data analysis. 17th Edn., Addison-Wesley, Reading, Mass., ISBN-10: 0201076160, pp: 688. 